\title{
Respostas fisiológicas de ovinos alimentados com genótipos de palma forrageira
}

\author{
[Physiological responses of sheep fed with spineless cactus genotypes]
}

\section{"Artigo Científico/Scientific Article"}

\author{
Tomás Guilherme Pereira da Silva*, Levi Auto Lopes, Francisco Fernando Ramos de Carvalho, \\ Ângela Maria Vieira Batista, Adriana Guim, Júlio Cézar dos Santos Nascimento, \\ José Francisco da Silva Neto
}

Departamento de Zootecnia, Universidade Federal Rural de Pernambuco, Recife-PE, Brasil.

*Autor para correspondência/Corresponding author: E-mail: tomas-g@ hotmail.com

\begin{abstract}
Resumo
Objetivou-se avaliar parâmetros fisiológicos de ovinos submetidos a dietas à base de palma forrageira e o grau de correlação entre estas variáveis e a ingestão de nutrientes digestíveis totais. Foram utilizados 36 cordeiros, com peso corporal inicial de $22.0 \pm 2.90 \mathrm{~kg}$, idade média de seis meses, distribuídos em delineamento inteiramente casualizado, com três tratamentos e 12 repetições. O período experimental foi de 86 dias, sendo fornecida uma dieta base (controle) e dietas nas quais o feno de capim Tifton foi parcialmente substituído por palma miúda ou palma orelha de elefante mexicana. No $80^{\circ}$ dia do período experimental, os animais foram submetidos à avaliação de respostas fisiológicas: frequência respiratória e cardíaca, temperatura retal e superficial, sendo esses dados coletados nos turnos da manhã e da tarde. Não houve interação significativa entre os fatores 'dietas' e 'turno', assim como não foi observado efeito das dietas sobre os parâmetros fisiológicos. Os valores de frequência respiratória e a temperatura superficial foram maiores no turno da manhã $\left(98,73 \pm 32,30\right.$ respirações por minuto e $33,83^{\circ} \mathrm{C} \pm 0,81$, respectivamente) em relação ao turno da tarde $\left(78,79 \pm 24,53\right.$ respirações por minuto e $32,53^{\circ} \mathrm{C} \pm 0,89$, respectivamente). Observaram-se significativos coeficientes de correlação positiva entre o consumo de energia e a frequência respiratória nos animais que receberam a dieta controle, no turno da manhã, e entre o consumo de energia e a temperatura corporal, no turno da tarde. Existe correlação positiva de moderada a forte magnitude entre o consumo de nutrientes digestíveis totais e a frequência respiratória e temperatura corporal de ovinos submetidos à dieta sem palma forrageira.
\end{abstract}

Palavras-chave: cactáceas; cordeiros; energia; homeostase; incremento calórico.

\begin{abstract}
The aim of this study was to evaluate physiological responses of sheep fed diets based on spineless cactus and the degree of correlation between these variables and the intake of total digestible nutrients. A total of 36 lambs, aged approximately six months and with initial body weight of $22.0 \pm 2.90 \mathrm{~kg}$ were used, distributed in a completely randomized design, with three treatments and twelve repetitions. The experiment lasted 86 days, with a base diet (control) and diets in which part of Tifton hay was replaced by miuda spineless cactus or orelha de elefante mexicana spineless cactus. On the 80th day of the experimental period, the physiological responses of the animals were evaluated: respiratory and heart rate, rectal and superficial temperature, and these data were collected in the morning and afternoon shifts. There was no significant interaction between the 'diets' and 'shift' factors, as no effect of the diets on the physiological parameters was observed. The values of respiratory rate and surface temperature were higher in the morning shift $(98.73 \pm 32.30$ breaths per minute and $33.83 \pm 0.81$, respectively) in relation to the afternoon shift ( $78.79 \pm 24.53$ breaths per minute and $32.53 \pm 0.89$, respectively). Significant positive correlation coefficients were observed between energy intake and respiratory rate in animals that received the control diet in the morning shift, as well as between energy intake and body temperature in the afternoon shift. There is a positive correlation of moderate to strong magnitude between the intake of total digestible nutrients and the respiratory rate and body temperature of sheep submitted to the diet without spineless cactus.
\end{abstract}

Keywords: cactaceae; caloric increase; energy; homeostasis; lambs. 


\section{Introdução}

Fatores ambientais, como temperatura e umidade relativa do ar, precipitação pluviométrica e velocidade dos ventos, exercem efeitos diretos e indiretos sobre a produção animal, acarretando modificações na produtividade. Alterações significativas nestes parâmetros ambientais comprometem a homeostase do animal, provocando assim mudanças em diversas variáveis fisiológicas e, por conseguinte, em índices de produção e reprodução (Neiva et al., 2004; Souza et al., 2010).

Nessa perspectiva, além do estresse propiciado pelo ambiente no qual os animais estão sendo criados, a dieta também influencia a produção de calor nos ruminantes, de modo que tanto a quantidade quanto a qualidade dos alimentos interferem na produção de calor endógeno, com consequente variação nos parâmetros fisiológicos (Baccari Junior, 2001). Nesse cenário, a palma forrageira (Opuntia ou Nopalea), que configura um importante recurso alimentar utilizado em regiões áridas e semiáridas do mundo, destaca-se por apresentar elevados teores de nutrientes digestíveis totais (energia) e carboidratos não fibrosos, bem como alto conteúdo hídrico (Batista et al., 2003; Costa et al., 2012; Pessoa et al., 2020), o que poderia interferir no consumo alimentar e promover modificações em variáveis fisiológicas dos animais que a recebem como ingrediente dietético.

Segundo Correia et al. (2012), recursos alimentares não convencionais devem ser avaliados em relação aos efeitos adversos ou benéficos que eventualmente podem promover sobre os animais que os ingerem, sobretudo em regiões tropicais. Assim, alterações na homeostase de pequenos ruminantes têm sido quantificadas mediante análise de variáveis fisiológicas, como temperatura retal e corporal, frequência respiratória e cardíaca (Bezerra et al., 2011; Oliveira et al., 2013; Barros Junior et al., 2017).

Diante do exposto, objetivou-se avaliar o efeito do fornecimento de dietas à base de genótipos de palma forrageira resistentes à cochonilha do carmim (Dactylopius sp.) sobre parâmetros fisiológicos de ovinos, bem como analisar a correlação desses parâmetros com o consumo de nutrientes digestíveis totais.

\section{Material e Métodos}

O experimento foi conduzido no galpão de confinamento do Setor de Caprinos e Ovinos do
Departamento de Zootecnia (DZ) da Universidade Federal Rural de Pernambuco (UFRPE), localizada no Recife-PE ( $8^{\circ} 04^{\prime} 03^{\prime \prime}$ de latitude sul, 34 ${ }^{\circ} 55^{\prime} 00^{\prime \prime}$ de longitude oeste e altitude de $4 \mathrm{~m}$ ). O clima da região, de acordo com a classificação climática de Köppen adaptada ao Brasil (Alvares et al., 2013), é do tipo 'AM'. A região tem uma precipitação média anual de $1804 \mathrm{~mm}$, dos quais 227,98 $\mathrm{mm}$ ocorrem entre maio e julho, quando há temperatura média máxima de $28,5^{\circ} \mathrm{C}$ (INMET, 2017).

Foram utilizados 36 cordeiros sem padrão racial definido, machos, não castrados, com idade média de seis meses e peso corporal inicial médio de $23,0 \pm 3,11 \mathrm{~kg}$, os quais foram pesados, identificados com brincos, vacinados contra clostridioses e submetidos ao controle de endo e ectoparasitas. Os animais foram alojados em baias individuais, providas de comedouro e de bebedouro, sendo distribuídos em delineamento inteiramente casualizado (DIC), com três tratamentos e 12 repetições.

Os tratamentos experimentais consistiram de uma dieta base (composta por feno de capim Tifton, fubá de milho, farelo de soja, ureia pecuária e sal mineralizado) e de mais duas dietas nas quais o feno de capim Tifton foi parcialmente $(75 \%)$ substituído pelos genótipos de palma forrageira (miúda - Nopalea cochenillifera Salm Dyck ou orelha de elefante mexicana - Opuntia stricta Haw), com proporção volumoso:concentrado de 60:40 (Tabela 1).

O período experimental compreendeu 86 dias, sendo 30 dias destinados à adaptação dos animais às condições experimentais (instalações e manejo) e 56 dias para coleta de amostras. O arraçoamento foi realizado duas vezes ao dia $(08 \mathrm{~h}$ e 15h), na forma de mistura completa, com água $a d$ libitum. Durante todo o período experimental, os alimentos fornecidos e as sobras foram pesados para mensuração do consumo alimentar (por animal/dia). O consumo de nutrientes digestíveis totais (NDT) usado no presente estudo foi obtido por Lopes et al. (2020).

No $80^{\circ}$ dia do período experimental, os animais foram submetidos à avaliação de respostas fisiológicas: frequência respiratória, frequência cardíaca, temperatura retal e temperatura superficial, sendo esses dados coletados nos turnos da manhã (entre 08h e 09h) e da tarde (entre $15 \mathrm{~h}$ e 16h). A frequência respiratória foi obtida através de movimentos do flanco por minuto, com o auxílio de cronômetro digital por período de 15 segundos e o resultado multiplicado por quatro para obtenção 
em minutos. A frequência cardíaca foi obtida através de estetoscópio posicionado diretamente na região torácica esquerda. A temperatura retal foi aferida através de termômetro clínico digital inserido no reto, em contato direto com a mucosa, por cerca de 1 minuto. A temperatura superficial foi obtida com o auxílio de termômetro infravermelho digital direcionado para região do dorso e dos membros, para obtenção de uma média por animal. Com o intuito de registrar a temperatura $\left({ }^{\circ} \mathrm{C}\right)$ e a umidade relativa do ar $(\%)$ foi instalado um termohigrômetro no centro do galpão experimental, na altura dos animais.
O programa SAS (1996) foi utilizado para a realização da análise estatística dos dados, sendo estes submetidos à análise de variância e as médias comparadas pelo teste de Tukey ao nível de 5\%, analisados em esquema de parcelas subdivididas. Para a interpretação da magnitude das correlações foi adotada a seguinte classificação dos coeficientes de correlação: < 0,4 (fraca magnitude); $>0,4$ a $<0,5$ (moderada magnitude) e $>0,5$ (forte magnitude). O software Minitab versão 17 (2014) foi utilizado para a realização da análise de correlação de Pearson entre as variáveis, adotando-se 5\% como nível de significância.

Tabela 1. Proporção dos ingredientes e composição nutricional das dietas experimentais.

\begin{tabular}{|c|c|c|c|}
\hline \multirow[t]{2}{*}{ Ingredientes } & \multicolumn{3}{|c|}{$\left(\mathrm{g} \mathrm{kg}^{-1} \text { de } \mathrm{MS}\right)^{*}$} \\
\hline & Controle & Miúda & OEM $^{* *}$ \\
\hline Feno de Tifton-85 & 600 & 150 & 150 \\
\hline Palma miúda & 0 & 450 & 0 \\
\hline Palma OEM ${ }^{* *}$ & 0 & 0 & 450 \\
\hline Fubá de milho & 270 & 271 & 273 \\
\hline Farelo de soja & 110 & 100 & 100 \\
\hline Ureia pecuária & 5 & 14 & 12 \\
\hline Sal mineral & 15 & 15 & 15 \\
\hline Total & 100 & 100 & 100 \\
\hline \multicolumn{4}{|c|}{ Composição química ( $\left.\mathrm{g} \mathrm{kg}^{-1} \mathrm{de} \mathrm{MS}\right)$} \\
\hline Matéria seca $\left(\mathrm{MN}^{* * *}\right)$ & 890,8 & 234,8 & 190,3 \\
\hline Matéria orgânica & 924,0 & 904,2 & 895,3 \\
\hline Matéria mineral & 76,0 & 95,8 & 104,7 \\
\hline Proteína bruta & 142,1 & 141,8 & 143,2 \\
\hline Extrato etéreo & 25,6 & 21,5 & 23,4 \\
\hline $\mathrm{FDNcp}^{1}$ & 456,1 & 267,4 & 243,1 \\
\hline Carboidratos não fibrosos & 300,2 & 473,4 & 485,8 \\
\hline Carboidratos totais & 756,3 & 740,8 & 728,8 \\
\hline Nutrientes digestíveis totais & 648,2 & 709,8 & 632,7 \\
\hline
\end{tabular}

\section{Resultados e Discussão}

Não foi observada interação significativa entre os fatores (dieta e turno), bem como não houve efeito dietético sobre as variáveis avaliadas (Tabela 2).

Por outro lado, a frequência respiratória e a temperatura superficial foram maiores no turno da manhã $(98,73 \pm 32,30$ respirações por minuto e $33,83^{\circ} \mathrm{C} \pm 0,81$, respectivamente) quando comparadas ao turno da tarde $(78,79 \pm 24,53$ respirações por minuto e $32,53^{\circ} \mathrm{C} \pm 0,89$, respectivamente), o que pode ser explicado pela maior temperatura ambiental e maior umidade relativa do ar no período matutino $\left(32,5^{\circ} \mathrm{C}\right.$ e $70 \%$, respectivamente) em relação ao período da tarde $\left(29,6^{\circ} \mathrm{C}\right.$ e $59 \%$, respectivamente). Todos os valores registrados para frequência cardíaca estavam acima da normalidade para ovinos (Tabela 2), que deve oscilar entre 70 e 80 batimentos cardíacos por minuto.

É relevante salientar que, independente da dieta e do turno, os animais demonstraram valores de frequência respiratória acima da faixa fisiológica citada por Ribeiro et al. (2008), que é de 20 a 36 movimentos por minuto, indicando que os ovinos estavam sob condições termicamente estressantes. A frequência respiratória representa um interessante indicador da situação de estresse 
térmico em ovinos, que usam esse mecanismo como dissipador de calor, além de servir para mensurar a severidade do estresse calórico que, ao atingir 70 a 100 movimentos por minuto, o animal passa a ser considerado estressado (Silanikove, 2000). Animais com respiração acelerada podem diminuir a ingestão de alimentos e gastar energia que poderia ser utilizada em processos metabólicos e produtivos (Vieira et al., 2016).
De acordo com Cunningham (2004), a temperatura retal considerada normal em ovinos varia de 38,5 a $39,9^{\circ} \mathrm{C}$ e diversos fatores podem causar variações na temperatura corporal, dentre eles idade, sexo, estação do ano, período do dia, exercício, ingestão e digestão de alimentos. Assim, os valores da temperatura retal estiveram dentro da faixa de normalidade preconizada para a espécie.

Tabela 2. Parâmetros fisiológicos de ovinos submetidos a dietas contendo genótipos de palma forrageira.

\begin{tabular}{lcccccccc}
\hline \multirow{2}{*}{ Indicador } & \multicolumn{3}{c}{ Tratamento $(\mathbf{T r})$} & \multicolumn{2}{c}{ Turno $(\mathbf{T u})$} & \multicolumn{3}{c}{ P-Valor } \\
\cline { 2 - 8 } & Controle & Miúda & OEM $^{1}$ & Manhã & Tarde & Tr & Tu & $\mathrm{Tr} * \mathrm{Tu}$ \\
\hline $\mathrm{FC}^{2}$ & $119,63 \pm 17,1$ & $121,66 \pm 11,97$ & $121,66 \pm 12,70$ & $124 \pm 1,23$ & $117,97 \pm 13,97$ & 0,853 & 0,080 & 0,853 \\
$\mathrm{FR}^{3}$ & $82,54 \pm 26,32$ & $98,33 \pm 32,78$ & $84,91 \pm 28,11$ & $98,73 \pm 32,30 \mathrm{a}$ & $78,79 \pm 24,53 \mathrm{~b}$ & 0,111 & 0,005 & 0,497 \\
$\mathrm{TR}^{4}$ & $38,68 \pm 2,21$ & $39,23 \pm 0,35$ & $39,04 \pm 0,24$ & $39,16 \pm 0,26$ & $38,81 \pm 1,76$ & 0,327 & 0,273 & 0,362 \\
$\mathrm{TC}^{5}$ & $33,43 \pm 0,99$ & $33,22 \pm 1,13$ & $32,89 \pm 1,05$ & $33,83 \pm 0,81 \mathrm{a}$ & $32,53 \pm 0,89 \mathrm{~b}$ & 0,095 & $<0.000$ & 0,337 \\
\hline
\end{tabular}

Médias seguidas por letras minúsculas diferentes na linha para cada fator são estatisticamente diferentes pelo teste Tukey ao nível de 5\% de significância. ${ }^{1} \mathrm{OEM}$ : palma orelha de elefante mexicana; ${ }^{2} \mathrm{FC}$ : frequência cardíaca; ${ }^{3} \mathrm{FR}$ : frequência respiratória; ${ }^{4} \mathrm{TR}$ : temperatura retal; ${ }^{5} \mathrm{TC}$ : temperatura corporal.

Verificou-se que as variáveis frequência respiratória e temperatura corporal correlacionamse positiva e significativamente com a ingestão de nutrientes digestíveis totais apenas nos animais que receberam a dieta controle, nos dados obtidos pela manhã e pela tarde, respectivamente (Tabela 3).

Esses resultados podem ser explicados pelo fato da dieta controle ser composta por $60 \%$ de feno de Tifton como volumoso, enquanto que nas demais dietas havia $75 \%$ de substituição do feno por um dos genótipos de palma forrageira (miúda ou orelha de elefante mexicana). Dietas ricas em alimentos volumosos, principalmente aqueles com alto teor fibroso, tendem a promover maior incremento calórico no processo digestivo. Assim, a maior produção de calor corporal gerado pelo consumo da dieta sem palma forrageira (controle) aumentou a temperatura dos ovinos e pode ter desencadeado uma maior necessidade dos animais em relação à utilização do mecanismo de elevação da frequência respiratória na tentativa de dissipar calor do organismo.

Adicionalmente, a maior produção de calor corpóreo propiciado pelo consumo de alimentos volumosos pode ser associada ao maior tempo de retenção (menor taxa de passagem) desses alimentos no trato gastrintestinal, assim como a maior dificuldade de digestão. Para Nobre et al. (2013), alimentos ricos em fibra promovem maior incremento calórico no organismo de animais ruminantes devido às características da fermentação que sofrem no processo digestivo.

$\mathrm{O}$ maior consumo de água (via alimentos e total) por ovinos alimentados com dietas contendo palma forrageira tem sido registrado em diversas investigações científicas (Costa et al., 2012; Andrade et al., 2016; Cardoso et al., 2019; Gouveia et al., 2019), podendo a maior ingestão hídrica auxiliar na termorregulação dos animais. É oportuno mencionar que, como reportado por Lopes et al. (2020), em trabalho de pesquisa associado ao presente estudo, não houve impacto dietético sobre parâmetros produtivos, como ganho de peso.

\section{Conclusão}

A utilização da palma forrageira na dieta de ovinos não provoca prejuízos aos parâmetros fisiológicos, sendo as condições climáticas as responsáveis por aumentarem a frequência respiratória e a temperatura superficial dos animais no turno matutino. Existe correlação positiva de moderada a forte magnitude entre o consumo de nutrientes digestíveis totais e a frequência respiratória e temperatura corporal de ovinos alimentados com feno de capim Tifton como volumoso exclusivo. 
Tabela 3. Coeficientes de correlação de Pearson e valor da probabilidade $(\mathrm{P})$ entre variáveis fisiológicas e consumo de nutrientes digestíveis totais (CNDT) de ovinos alimentados com dietas à base de palma forrageira, nos turnos da manhã e da tarde

\begin{tabular}{|c|c|c|}
\hline \multirow[b]{2}{*}{ Variável } & \multicolumn{2}{|c|}{ CNDT (g/dia) } \\
\hline & Coeficiente de correlação & Valor de $\mathbf{P}$ \\
\hline Manhã & \multicolumn{2}{|c|}{ Controle } \\
\hline $\mathrm{TC}^{1}\left({ }^{\circ} \mathrm{C}\right)$ & 0,30678 & 0,3321 \\
\hline $\mathrm{TR}^{2}\left({ }^{\circ} \mathrm{C}\right)$ & $-0,02166$ & 0,9467 \\
\hline $\mathrm{FC}^{3}$ (batimentos/minuto) & 0,41121 & 0,1842 \\
\hline \multirow[t]{2}{*}{$\mathrm{FR}^{4}$ (respirações/minuto) } & 0,58115 & 0,0475 \\
\hline & \multicolumn{2}{|c|}{ Miúda } \\
\hline $\mathrm{TC}^{1}\left({ }^{\circ} \mathrm{C}\right)$ & 0,02317 & 0,9430 \\
\hline $\mathrm{TR}^{2}\left({ }^{\circ} \mathrm{C}\right)$ & 0,23582 & 0,4606 \\
\hline $\mathrm{FC}^{3}$ (batimentos/minuto) & 0,00765 & 0,9812 \\
\hline \multirow[t]{2}{*}{$\mathrm{FR}^{4}$ (respirações/minuto) } & 0,04699 & 0,8847 \\
\hline & \multicolumn{2}{|c|}{ OEM* } \\
\hline $\mathrm{TC}^{1}\left({ }^{\circ} \mathrm{C}\right)$ & 0,12759 & 0,7254 \\
\hline $\mathrm{TR}^{2}\left({ }^{\circ} \mathrm{C}\right)$ & $-0,07643$ & 0,8338 \\
\hline $\mathrm{FC}^{3}$ (batimentos/minuto) & 0,16404 & 0,6507 \\
\hline $\mathrm{FR}^{4}$ (respirações/minuto) & $-0,33027$ & 0,3513 \\
\hline Tarde & \multicolumn{2}{|c|}{ Controle } \\
\hline $\mathrm{TC}^{1}\left({ }^{\circ} \mathrm{C}\right)$ & 0,59559 & $\mathbf{0 , 0 5 3 2}$ \\
\hline $\mathrm{TR}^{2}\left({ }^{\circ} \mathrm{C}\right)$ & 0,13475 & 0,6928 \\
\hline $\mathrm{FC}^{3}$ (batimentos/minuto) & $-0,28742$ & 0,3914 \\
\hline \multirow[t]{2}{*}{$\mathrm{FR}^{4}$ (respirações/minuto) } & 0,39511 & 0,2291 \\
\hline & \multicolumn{2}{|c|}{ Miúda } \\
\hline $\mathrm{TC}^{1}\left({ }^{\circ} \mathrm{C}\right)$ & $-0,25935$ & 0,4156 \\
\hline $\mathrm{TR}^{2}\left({ }^{\circ} \mathrm{C}\right)$ & 0,11145 & 0,7302 \\
\hline $\mathrm{FC}^{3}$ (batimentos/minuto) & 0,00303 & 0,9925 \\
\hline \multirow[t]{2}{*}{$\mathrm{FR}^{4}$ (respirações/minuto) } & 0,24554 & 0,4418 \\
\hline & \multicolumn{2}{|c|}{ OEM $^{*}$} \\
\hline $\mathrm{TC}^{1}\left({ }^{\circ} \mathrm{C}\right)$ & 0,43494 & 0,1576 \\
\hline $\mathrm{TR}^{2}\left({ }^{\circ} \mathrm{C}\right)$ & 0,00951 & 0,9766 \\
\hline $\mathrm{FC}^{3}$ (batimentos/minuto) & 0,53767 & 0,0714 \\
\hline $\mathrm{FR}^{4}$ (respirações/minuto) & 0,55185 & 0,0629 \\
\hline
\end{tabular}

${ }^{1} \mathrm{TC}$ : temperatura corporal; ${ }^{2} \mathrm{TR}$ : temperatura retal; ${ }^{3} \mathrm{FC}$ : frequência cardíaca; ${ }^{4} \mathrm{FR}$ : frequência respiratória; ${ }^{*} \mathrm{OEM}$ : palma orelha de elefante mexicana.

\section{Conflito de Interesse}

Os autores declaram não existir conflito de interesse.

\section{Comitê de Ética}

O projeto de pesquisa foi aprovado pelo Comitê de Ética da Universidade Federal Rural de Pernambuco, sob o número 142/2018.

\section{Agradecimentos}

Esta pesquisa foi apoiada pelo Conselho Nacional de Desenvolvimento Científico e Tecnológico $(\mathrm{CNPq})$, pela Fundação de Amparo à Ciência e Tecnologia do Estado de Pernambuco (FACEPE). Este estudo foi financiado em parte pela Coordenação de Aperfeiçoamento de Pessoal de Nível Superior (CAPES) - Código Financeiro 001 .

\section{Referências}

Alvares, C.A; Stape, J.L.; Sentelhas, P.C.; de Moraes Gonçalves, J.L.; Sparovek, G. Köppen's climate classification map for Brazil. Meteorologische Zeitschrift, 22(6): 711-728, 2013.

Andrade, S.F.J.; Batista, Â.M.V.; Carvalho, F.F.R.; Lucena, R.B.; Andrade, R.P.X.; Lima Júnior, D.M. Fresh or dehydrated spineless cactus in diets for lambs. Acta Scientiarum. Animal Sciences, 38(2): 155-161, 2016.

Baccari Junior, F. Manejo ambiental da vaca leiteira em climas quentes. Londrina, PR: UEL, 2001. 142 p.

Barros Junior, C.P.; Sousa Júnior, S.C.; Campelo, J.E.G.; Azevedo, D.M.M.R.; Carvalho, G.M.C.; Sousa, P.H.A.A. Avaliação de parâmetros fisiológicos em diferentes raças de caprinos na Região Nordeste brasileira. REDVET Revista Electrónica de Veterinaria, 19(1): 1$11,2017$. 
Batista, A.M.V.; Mustafa, A.F.; McAllister, T.; Wang, Y.; Soita, H.; McKinnon, J.J. Effects of variety on chemical composition in situ nutrient disappearance and in vitro gas production of spineless cacti. Journal of the Science of Food and Agriculture, 83(5): 440-445, 2003.

Bezerra, W.M.A.X.; Souza, B.B.; Sousa, W.H.; Cunha, M.G.G.; Benicio, M.A. Comportamento fisiológico de diferentes grupos genéticos de ovinos criados no semiárido paraibano. Revista Caatinga, 24(1): 130-136, 2011.

Cardoso, D.B., Carvalho, F.F.R., Medeiros, G.R., Guim, A., Cabral, A.M.D., Véras, R.M.L., Santos, K.C., Dantas, L.C.N.; Nascimento, A.G.O. Levels of inclusion of spineless cactus (Nopalea cochenillifera Salm Dyck) in the diet of lambs. Animal Feed Science and Technology. 247(1): 23-31, 2019.

Correia, B.R.; Oliveira, R.L.; Jaeger, S.M.P.L.; Bagaldo, A.R.; Carvalho, G.G.P.; Oliveira, G.J.C.; Lima, F.H.S.; Oliveira, P.A. Comportamento ingestivo e parâmetros fisiológicos de novilhos alimentados com tortas do biodiesel. Archivos de Zootecnia, 61(233): 79-89, 2012.

Costa, R.G.; Treviño, I.H.; Medeiros, G.R.; Medeiros, A.N.; Pinto, T.F.; Oliveira, R.L. Effects of replacing corn with cactus pear (Opuntia ficus indica Mill) on the performance of Santa Inês lambs. Small Ruminant Research, 102: 13-17, 2012.

Cunningham, J.G. Tratado de fisiologia veterinária. $3^{\mathrm{a}}$ ed. Rio de Janeiro: GuanabaraKoogan, 2004. 596 p.

Gouveia, L.N.F.; Soares, P.C.; Moura, M.S.C.; Silva, T.G.P.; Silva Neto, I.F.; Guimarães, D.N.A.; Cardoso, D.B.; Batista, Â.M.V.; Carvalho, F.F.R. Metabolic profile and renal function of lambs fed with maniçoba hay replacement by spineless cactus. Revista Agrária Acadêmica, 2(4): 41-51, 2019.

INMET - Instituto Nacional de Meteorologia, 2017. Parâmetros meteorológicos de Recife, Pernambuco. Disponível em: <http://www.inmet.gov.br/>. Acesso em: 05 dez. 2017.

Lopes, L.A.; Ferreira, M.A.; Batista, Â.M.V.; Maciel, M.V.; Barbosa, R.A.; Munhame, J.A.; Silva, T.G.P.; Cardoso, D.B.; Véras, A.S.C.; Carvalho, F.F.R. Intake, digestibility, and performance of lambs fed spineless cactus $\mathrm{cv}$. Orelha de Elefante Mexicana. AsianAustralasian Journal of Animal Sciences, 33(8): 1284-1291, 2020.

Minitab. Minitab Quality Companion. 2014:
Disponível em: <http://www.minitab.com>. Acesso em: 12 dez. 2018.

Neiva, J.N.M.; Teixeira, M.; Turco, S.H.N. Efeito do estresse climático sobre os parâmetros produtivos e fisiológicos de ovinos Santa Inês mantidos em confinamento na região litorânea do Nordeste do Brasil. Revista Brasileira de Zootecnia, 33(3): 668-678, 2004.

Nobre, I.S; Souza, B.B.; Marques, B.A.A.; Batista, N.L. Efeito de diferentes níveis de concentrado e inclusão de gordura protegida na dieta sobre o desempenho produtivo e termorregulação de ovinos. Agropecuária Científica no SemiÁrido, 9(2): 14-20, 2013.

Oliveira, F.A.; Turco, S.N.H.; Borges, I.; Clemente, C.A.A.; Nascimento, T.V.C.; Loiola Filho, J.B. Parâmetros fisiológicos de ovinos Santa Inês submetidos a sombreamento com tela de polipropileno. Revista Brasileira de Engenharia Agrícola e Ambiental, 17(9): 1014-1019, 2013.

Pessoa, D.V.; Andrade, A.P.; Magalhães, A.L.R.; Teodoro, A.L.; Santos, D.C.; Araújo, G.G.L.; Medeiros, A.N.; Nascimento, D.B.; Valença, R.L.; Cardoso, D.B. Forage cactus of the genus Opuntia in different with the phenological phase: nutritional value. Journal of Arid Environments, 181: 1-8, 2020.

Ribeiro, N.L.; Furtado, D.A.; Medeiros, A.N.; Ribeiro, M.N.; Silva, R.C.B.; Souza, C.M.S. Avaliação dos índices de conforto térmico, parâmetros fisiológicos e gradiente térmico de ovinos nativos. Revista Engenharia Agrícola, 28(4): 614-623, 2008.

Silanikove, N. Effects of heat stress on the welfare of extensively managed domestic ruminants. Livestock Production Science, 67:1-18, 2000.

Souza, B.B.; Lopes, J.J.; Roberto, J.V.B.; Silva, A.M.A.; Silva, E.M.N.; Silva, G.A. Efeito do ambiente sobre as respostas fisiológicas de Caprinos Saanen e mestiços 1/2Saanen $+1 / 2$ Boer no semiárido Paraibano. Agropecuária Científica no Semi-Árido, 6(2): 47-51, 2010.

SAS. Statistical Analysis System Institute - SAS Institute. User's guide: statistics. Versão 6. 12. Cary: North Carolina State University, 1996.

Vieira, M.M.M.; Furtado, F.M.V.; Cândido, M.J.D.; Barbosa Filho, J.A.D.; Cavalcante, A.C.R.; Magalhães, J.A.; Costa, N.L. Aspectos fisiológicos e bioclimáticos de caprinos nas regiões semiáridas. PUBVET, 10(5): 356-369, 2016.

MINITAB 17: Statistical software. 